ARTICLE

Received 17 Dec 2013 | Accepted 7 Mar 2014 | Published 20 May $2014 \quad$ DOl: 10.1038/ncomms4593

\title{
Terminal supraparticle assemblies from similarly charged protein molecules and nanoparticles
}

Jai II Park ${ }^{1, \star, \dagger}$, Trung Dac Nguyen ${ }^{1, \star}$, Gleiciani de Queirós Silveira', Joong Hwan Bahng ${ }^{2}$, Sudhanshu Srivastava ${ }^{1}$, Gongpu Zhao ${ }^{3}$, Kai Sun ${ }^{4}$, Peijun Zhang ${ }^{3}$, Sharon C. Glotzer ${ }^{1,2,5}$ \& Nicholas A. Kotov ${ }^{1,2,4,5}$

Self-assembly of proteins and inorganic nanoparticles into terminal assemblies makes possible a large family of uniformly sized hybrid colloids. These particles can be compared in terms of utility, versatility and multifunctionality to other known types of terminal assemblies. They are simple to make and offer theoretical tools for designing their structure and function. To demonstrate such assemblies, we combine cadmium telluride nanoparticles with cytochrome $\mathrm{C}$ protein and observe spontaneous formation of spherical supraparticles with a narrow size distribution. Such self-limiting behaviour originates from the competition between electrostatic repulsion and non-covalent attractive interactions. Experimental variation of supraparticle diameters for several assembly conditions matches predictions obtained in simulations. Similar to micelles, supraparticles can incorporate other biological components as exemplified by incorporation of nitrate reductase. Tight packing of nanoscale components enables effective charge and exciton transport in supraparticles and bionic combination of properties as demonstrated by enzymatic nitrate reduction initiated by light absorption in the nanoparticle.

\footnotetext{
${ }^{1}$ Departments of Chemical Engineering, University of Michigan, Ann Arbor, Michigan 48109, USA. ${ }^{2}$ Biomedical Engineering, University of Michigan, Ann Arbor, Michigan 48109, USA. ${ }^{3}$ Department of Structural Biology, University of Pittsburgh School of Medicine, Pittsburgh, Pennsylvania 15260, USA. ${ }^{4}$ Materials Science and Engineering, University of Michigan, Ann Arbor, Michigan 48109, USA. ${ }^{5}$ Biointerfaces Institute, University of Michigan, Ann Arbor, Michigan 48019, USA. * These authors contributed equally to this work. †Present address: Nuclear Chemistry Research Division, Korea Atomic Energy Research Institute (KAERI), Daejeon 305-353, Korea. Correspondence and requests for materials should be addressed to S.C.G. (email: sglotzer@umich.edu) or to N.A.K. (email: kotov@umich.edu).
} 
S elf-organization of semiconductor or metal nanoparticles (NPs) leads to nano- and microscale superstructures with geometries reminiscent of those produced by biological macromolecules ${ }^{1-7}$. Distinct parallels can be also made between assemblies of globular proteins and those made by $\mathrm{NPs}^{5-8}$. While many geometrical motifs for assemblies of proteins are known, those between NP and biomacromolecules are limited. They are represented predominantly by extended assemblies, that is, those without defined size requirements for some assembly directions. Extended NP assemblies produce polydisperse nano- and microscale structures and can be exemplified by templated NP adsorbates 9 , cocrystallized NP-protein superlattices ${ }^{7}$ and free-floating NP chains, sheets and ribbons of different lengths ${ }^{1,3,10}$. Whereas terminal assemblies are those that can be formed only with inherent size restrictions in all directions, for example, micelles, vesicles and viral capsids (Supplementary Note 1). Such systems are fundamentally and technologically attractive due to their uniformity, versatility and simplicity of preparation. Terminal assemblies are not known for hybrid NP-biomacromolecule systems. Covalent bioconjugates ${ }^{10,11}$, electrostatic complexes between single-protein molecules and $\mathrm{NPs}^{12,13}$, biomolecular coronas around $\mathrm{NPs}^{14}$ and similar structures ${ }^{15}$ display dimensional restrictions, but it is difficult to classify them as terminal assemblies for reasons of preparative methods, small number of particles, uniformity or stability. Finding a way to make hybrid nano-bio terminal assemblies would open the door to a new diverse family of colloids. Besides being a potential analytical ${ }^{13}$ and drug delivery too ${ }^{16}$, the scientific value of such systems will be the possibility to integrate biological functions of proteins with optical and electrical properties of metallic and semiconducting materials. They may also uncover unknown biological effects of NPs present in the environment ${ }^{17}$.

In this paper, we present a new type of protein-NP hybrid structures, known as supraparticles $(\mathrm{SPs})^{18}$, that spontaneously assemble under a variety of conditions from cadmium telluride (CdTe) NPs and cytochrome C (CytC). SPs represent a case of stable self-limited terminal assemblies made possible by the balance of attractive and repulsive forces between the building blocks that make them similar to other terminal assemblies. Applications of this research may include the realization of bionic assemblies with novel properties such as photoenzymatic activity $^{8,19,20}$, enhanced stability ${ }^{21}$ and potentially self-repair.

\section{Results}

Self-assembly of CdTe NPs and CytC. Positively charged $3.8 \pm 0.4 \mathrm{~nm}$ CdTe NPs, stabilized by 2-(dimethylamino) ethanethiol (DMAET), are known to self-assemble into microscale sheets ${ }^{3,22}$. Among the wide range of choices for a 'complementary' biomolecule, we choose CytC, a well-studied protein $3.1 \mathrm{~nm}$ in size and a dipole moment as high as $\sim 340$ Debyes (Supplementary Note 2 ) $^{23,24}$. CytC alone does not reveal a tendency to self-assemble in aqueous solution at $\mathrm{pH} \sim 7$. The isoelectric point of $\mathrm{CytC}$ is 11.0; therefore, it is positively charged over a wide $\mathrm{pH}$ range. The choice of a positively charged protein for combining with positively charged NPs seems, at first glance, counterintuitive in promoting self-assembly. Conventionally, electrostatic attraction between oppositely charged building blocks drives self-assembly (Supplementary Note 3$)^{9,12,25}$. However, as we will demonstrate below, counterbalancing electrostatic repulsion with intermolecular attractive interactions including dipolar, hydrogen bonding, hydrophobic and van der Waals (vdW) forces is a viable approach that leads to terminal structures. Besides surfactants, self-limitation due to the counterbalance of attractive-repulsive forces was theoretically predicted $^{26,27}$ and observed for NPs ${ }^{18,28}$ and electrified droplets of water. ${ }^{29}$

Six micromolar NP dispersion at $\mathrm{pH} \sim 5$ is mixed with $6 \mu \mathrm{M}$ CytC at $\mathrm{pH} \sim 7$. A stable NP-CytC dispersion with an electrokinetic potential $(\zeta)$ of $+30 \mathrm{mV}$ and $\mathrm{pH} \sim 5.3$ forms after $\sim 48-$ $72 \mathrm{~h}$. The presence of CytC strongly affects the NP assembly pattern ${ }^{3}$. Examination by scanning electron microscopy (SEM) and transmission electron microscopy (TEM) reveals the formation of uniformly sized, spherical SPs with a TEM diameter of $d_{\mathrm{TEM}}=94 \pm 5.6 \mathrm{~nm}$ (Fig. 1a,e, and Supplementary Fig. 1) instead of large sheets typical of DMAET-CdTe NPs ${ }^{3}$. Their size distribution is narrow with a s.d. less than $7 \%$. Dynamic light scattering (DLS) of 1:1 NP-CytC dispersion confirms the assembly of SPs with $d_{\mathrm{DLS}}=99 \pm 19.0 \mathrm{~nm}$ (Fig. 1f), which matches the diameter determined from SEM/TEM data. The difference between $d_{\mathrm{DLS}}$ and $d_{\mathrm{TEM}}$ indicates incorporation of water molecules in the SPs, as is observed in many proteins and their assemblies ${ }^{30}$. We find that self-assembly into spheres is specific to the pairing of NPs and CytC in a 1:1 starting ratio. When NPs are the dominant components, there is an increasing tendency to form 2D structures (Fig. 1b,c). When proteins are dominant, the size distribution increased and large irregular aggregates appeared (Fig. 1d, and Supplementary Fig. 2). Within them, 100-nm SPs are identifiable, indicating a specific tendency of CytC + DMAET-CdTe pairs to form spheres, unlike the diversity of other geometries observed before ${ }^{2-4,21}$.

The diameter of SPs shows limited dependence on the diameter of constitutive NPs. Specifically, a change in the NP average diameter from $2.7 \mathrm{~nm}$ to $7.2 \mathrm{~nm}$ results in a subtle change in diameter of SPs, remaining largely within experimental error (Table 1). However, when the average diameter of NPs is increased to $13.5 \mathrm{~nm}$, which is accompanied by a concomitant reduction in $\zeta$, the average diameter of SPs increased markedly to $970 \mathrm{~nm}$ (Table 1 and Supplementary Fig. 3). For most experiments, theoretical calculations and simulations reported here, we use the $3.8 \mathrm{~nm}$ of NPs.

We further examine the effect of ionic strength and temperature of assembly on the diameter of the SPs. The size of the SPs considerably decreases when the ionic strength of the assembly medium increases (Fig. 2a-d,e, Table 2). Meanwhile, the increase in temperature results in a slight increase in the SP average diameter (Fig. 2f, Table 3). These trends indicate the possibility to control the dimension of CytC-CdTe assemblies; they also suggest that 'phase diagrams' of SP morphologies can be constructed based on these media parameters.

The intermediate stages of the self-organization process indicate the path by which the SPs form. The appearance of particles with $d_{\mathrm{DLS}}=126 \pm 9.7, \quad 66 \pm 15.0, \quad 72 \pm 9.4$ and $100.6 \pm 17.3 \mathrm{~nm}$ are observed at $1,24,48$ and $72 \mathrm{~h}$, respectively (Supplementary Fig. 4). This is accompanied by a gradual disappearance of signals with $d_{\mathrm{DLS}}=5 \pm 0.4$ and $20 \pm 1.4 \mathrm{~nm}$, attributed to original NPs and early NP-CytC clusters, respectively. The initial increase and then decrease of $d_{\mathrm{DLS}}$ values show that large aggregates with a broad size distribution form quickly, and subsequently condense and stabilize in size by $72 \mathrm{~h}$. The sequence of the intermediate stages preceding the formation of stable SPs is confirmed by TEM images (Supplementary Fig. 5).

Supraparticle characterization. High-resolution transmission electron microscopy (HRTEM) of the assemblies indicates that they consist of reticulated electron-transparent and electrondense areas (Fig. 3a, and Supplementary Fig. 6) with a characteristic size of $\sim 5-7 \mathrm{~nm}$. Tomographic 3D reconstruction images of the spherical SPs show that these areas interpenetrate 
a

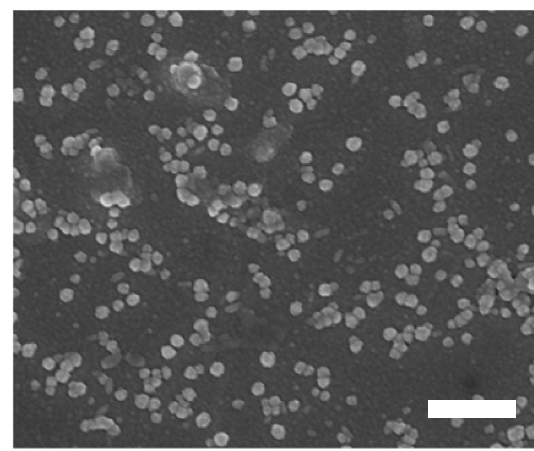

C

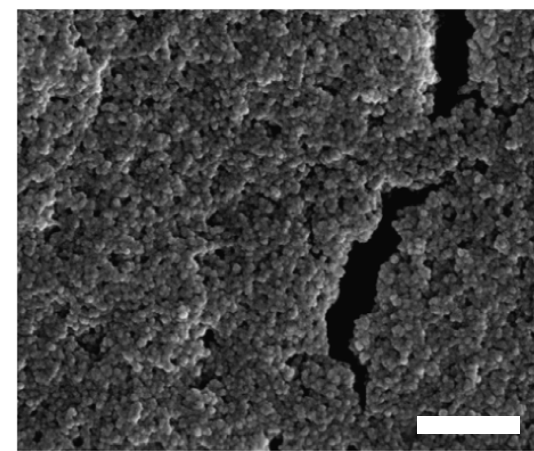

$\mathbf{e}$

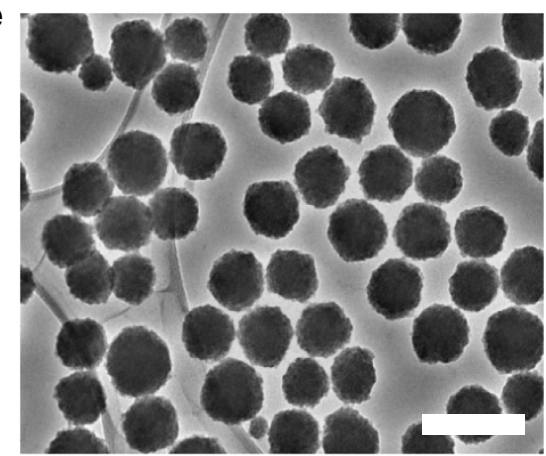

b

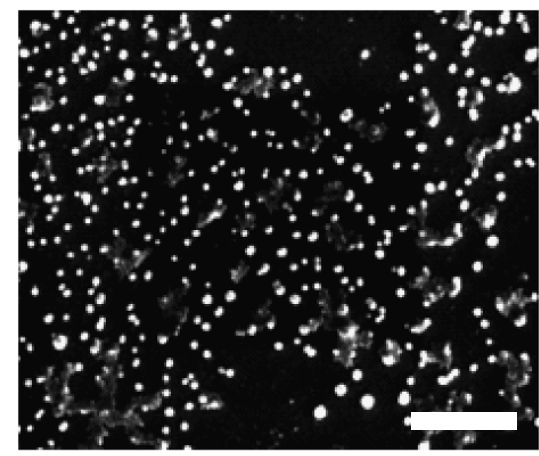

d

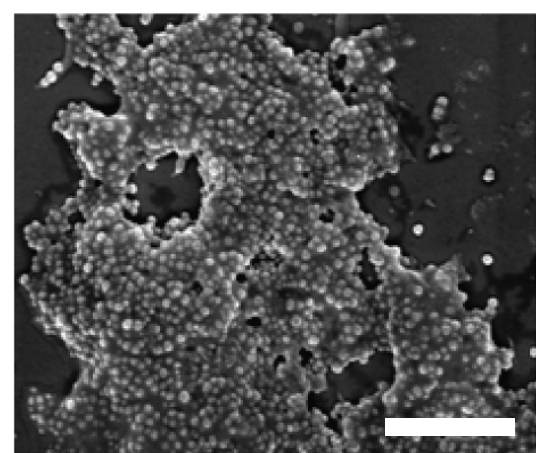

f

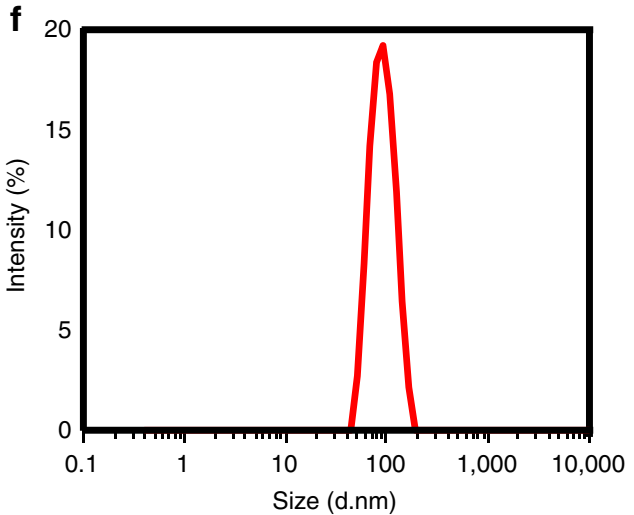

Figure 1 | Assembly of cadmium telluride nanoparticles(CdTe NPs) and cytochrome C (CytC). (a-d) Scanning electron miscroscopy (SEM) images of $\mathrm{CdTe} / \mathrm{CytC}$ assemblies with molar ratio of CdTe:CytC as 1:1 (a), 2:1 (b), and 6:1 (c). 1:6 (d). (e) Transmission electron microscopy (TEM) image of $\mathrm{CdTe} /$ CytC supraparticles (SPs). (f) Size distribution of the self-assembled SPs by dynamic light scattering (DLS). Scale bar, $500 \mathrm{~nm}(\mathbf{a}, \mathbf{c}), 1 \mu \mathrm{m}(\mathbf{b}, \mathbf{d})$, and $200 \mathrm{~nm}(\mathbf{e})$.

Table 1 | Dependence of SP diameter on the NP diameter.

\begin{tabular}{lccc}
$\begin{array}{l}\text { Diameter of } \\
\text { DMAET-CdTe } \\
\text { NPs }(\mathbf{n m})\end{array}$ & $\begin{array}{c}\text { Zeta } \\
\text { potential } \\
(\boldsymbol{\zeta})(\mathbf{m V})\end{array}$ & $\begin{array}{c}\text { Diameter of SPs } \\
\text { determined by } \\
\text { DLS } \boldsymbol{d}_{\text {DLs }}(\mathbf{n m})\end{array}$ & $\begin{array}{c}\text { Diameter of SPs } \\
\text { determined by } \\
\text { TEM } \boldsymbol{d}_{\text {TEM }}(\mathbf{n m})\end{array}$ \\
\hline $2.7 \pm 1.2$ & +28.9 & $98 \pm 22.3$ & $91 \pm 7.1$ \\
$3.8 \pm 0.4$ & +30.7 & $99 \pm 19.0$ & $94 \pm 5.6$ \\
$6.5 \pm 2.4$ & +32.6 & $109 \pm 13.5$ & $93 \pm 5.3$ \\
$6.7 \pm 1.1$ & +35.9 & $133 \pm 24.2$ & $95 \pm 3.4$ \\
$7.2 \pm 2.9$ & +38.4 & $147 \pm 20.8$ & $98 \pm 2.7$ \\
$13.5 \pm 2.3$ & +0.27 & $1,500 \pm 396$ & $970 \pm 61$ \\
\hline
\end{tabular}

DLS, dynamic light scattering; NP, nanoparticle; SP, supraparticle; TEM, transmission electron microscopy.

Statistical errors were calculated from multiple measurements on TEM grids and by DLS.

each other (Fig. 3b vii, viii, and Supplementary Movie 1 and 2) to form a network of tightly interconnected NP and CytC units. $\mathrm{X}$-ray energy dispersive spectroscopy (XEDS) confirms the presence of both NPs and CytC (Fig. 3c). Individual NPs in semiconductor-rich areas can be distinguished by its crystal lattice with periodicity of $0.38 \mathrm{~nm}$ expected for CdTe (111) lattice planes (Supplementary Fig. 6b). We do not observe any specific order within the network, although the tight packing with volume density of NPs considerably higher than percolation threshold suggests it to be bi-continuous. Note that the interconnectivity of the NPs, which is essential for charge and exciton transfer within SPs, is likely to be non-random here and is governed by modified percolation theory applied to selfassembly $^{31}$.

On the basis of the average volume of the SPs and the atomic ratio of $\mathrm{Cd}$ and $\mathrm{Fe}$, the total number of $\mathrm{CytC}$ and NPs in a SP can be estimated to be $3.6 \times 10^{3} \pm 7.5 \times 10^{2}$. The structures of individual SPs can be described by a schematic in Fig. $3 \mathrm{~d}$. We are interested in determining whether the process of SP formation (Supplementary Figs 4 and 5) causes a significant degree of structural changes in the softer component, that is, CytC molecules, when it assembles with the more rigid components, 
a

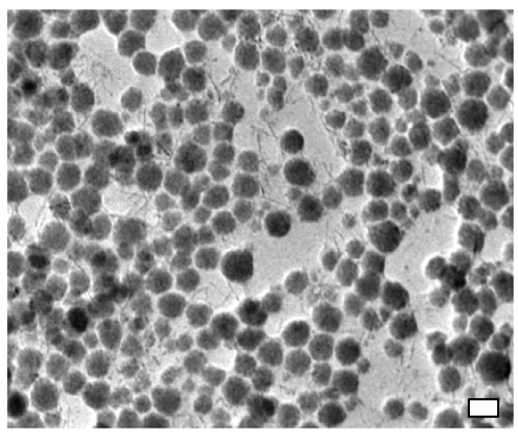

C

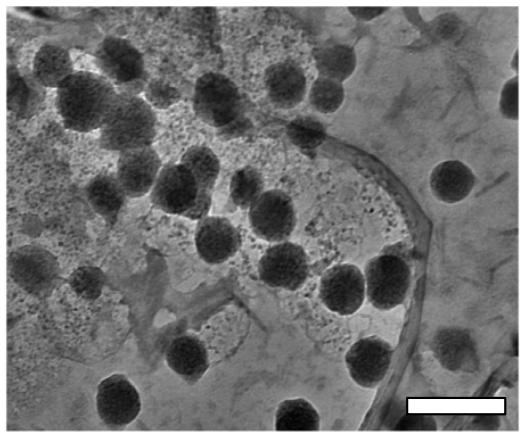

e

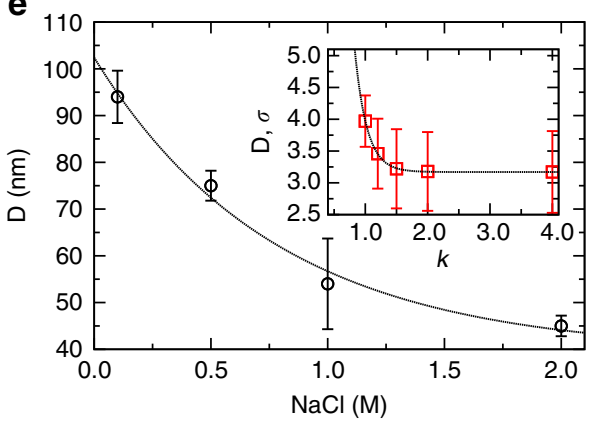

b

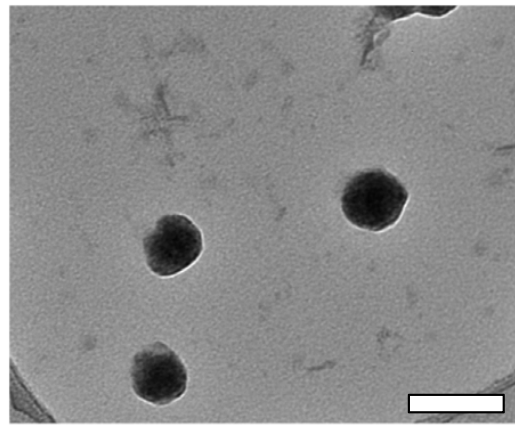

d
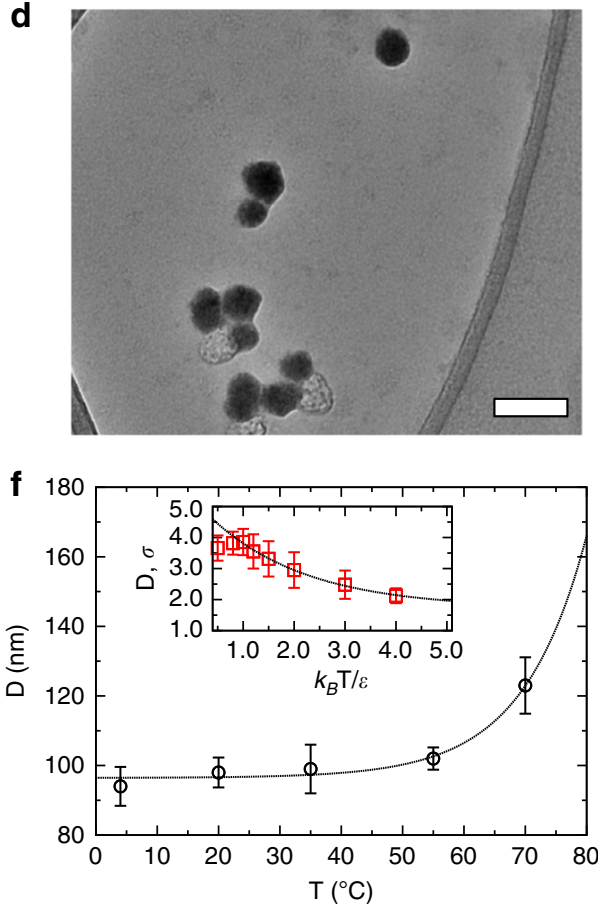

Figure 2 | Dependences of SP diameters on media parameters. (a-d) TEM images of SPs made in the solutions with (a) $0.1 \mathrm{M}$, (b) $0.5 \mathrm{M},(\mathbf{c}) 1.0 \mathrm{M}$ and (d) $2.0 \mathrm{M}$ of NaCl . Scale bars are $100 \mathrm{~nm}$. (e) Dependence of SP diameters on the $\mathrm{NaCl}$ concentration of $\mathrm{NaCl}$ and on inverse screening length in simulation (inset). (f) Temperature dependence of the SP diameters in experiment and in simulation (inset). The error bars in the experimental results $(\mathbf{e}, \mathbf{f})$ were obtained from the s.d. values from multiple experiments. Those in the simulation plots (insets in $\mathbf{e}$ and $\mathbf{f}$ ) are the corresponding s.d. of the SP size distribution.

Table 2 | Dependence of SP diameter on ionic strength.

\begin{tabular}{lcc}
$\begin{array}{l}\text { Concentration } \\
\text { of } \mathbf{N a C l}(\mathbf{M})\end{array}$ & $\begin{array}{c}\text { Diameter of SPs } \\
\text { determined by } \\
\mathbf{d}_{\mathbf{D L s}}(\mathbf{n m})\end{array}$ & $\begin{array}{c}\text { Diameter of SPs } \\
\text { determined by TEM } \\
\mathbf{d}_{\text {TEM }}(\mathbf{n m})\end{array}$ \\
\hline 0.1 & $99 \pm 19.2$ & $94 \pm 5.6$ \\
0.5 & $78 \pm 17.4$ & $75 \pm 3.2$ \\
1.0 & $61 \pm 12.2$ & $54 \pm 9.7$ \\
2.0 & $49 \pm 23.1$ & $45 \pm 2.2$ \\
\hline
\end{tabular}

DLS, dynamic light scattering; NP, nanoparticle; SP, supraparticle; TEM, transmission electron microscopy.

Statistical errors were calculated from multiple measurements on TEM grids and by DLS.

that is, CdTe NPs. During SP assembly, the $\pi-\pi^{*}$ peak of CytC at $409 \mathrm{~nm}$ (Soret band) red-shifts to $415 \mathrm{~nm}$, while the shoulder at $500 \mathrm{~nm}$ splits into two distinct peaks at $520 \mathrm{~nm}$ and $550 \mathrm{~nm}$ (Supplementary Fig. 7a). These transitions indicate a change in the oxidation state of the haem group in the protein from $\mathrm{Fe}^{+3}$ to
Table 3 | Dependence of SP diameter on the temperature.

\begin{tabular}{lcc}
$\begin{array}{l}\text { Temperature } \\
\left({ }^{\circ} \mathbf{C}\right)\end{array}$ & $\begin{array}{c}\text { Diameter of SPs } \\
\text { determined by DLS } \\
\mathbf{d}_{\mathbf{D L S}(\mathbf{n m})}\end{array}$ & $\begin{array}{c}\text { Diameter of SPs } \\
\text { determined by TEM } \\
\left.\mathbf{d}_{\mathbf{T E M}} \mathbf{( n m}\right)\end{array}$ \\
\hline 4 & $99 \pm 19.2$ & $94 \pm 5.6$ \\
20 & $105 \pm 21.3$ & $98 \pm 4.3$ \\
35 & $108 \pm 17.4$ & $99 \pm 7.0$ \\
55 & $111 \pm 18.1$ & $102 \pm 3.2$ \\
70 & $128 \pm 26.5$ & $123 \pm 8.1$ \\
\hline $\begin{array}{l}\text { DLS, dynamic light scattering; NP, nanoparticle; SP, supraparticle; TEM, transmission electron } \\
\text { microscopy. } \\
\text { Statistical errors were calculated from multiple measurements on TEM grids } \\
\text { and by DLS. }\end{array}$ \\
\hline
\end{tabular}

$\mathrm{Fe}^{+2}$ upon SP assembly ${ }^{32}$; from the extinction coefficient of the $\mathrm{Fe}^{+2} / \mathrm{Fe}^{+3}$ at $550 \mathrm{~nm}$, it is estimated that $\sim 77 \%$ of the iron atoms are present in their reduced state in the assembled SPs (Supplementary Note 4). 
a

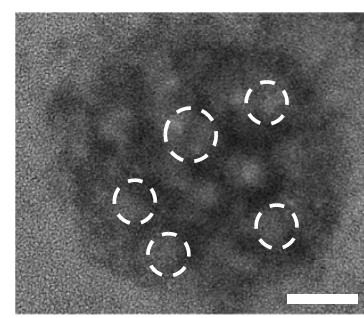

b

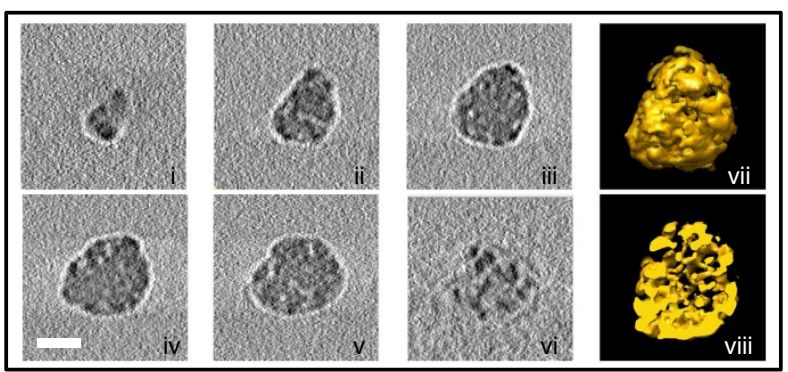

C

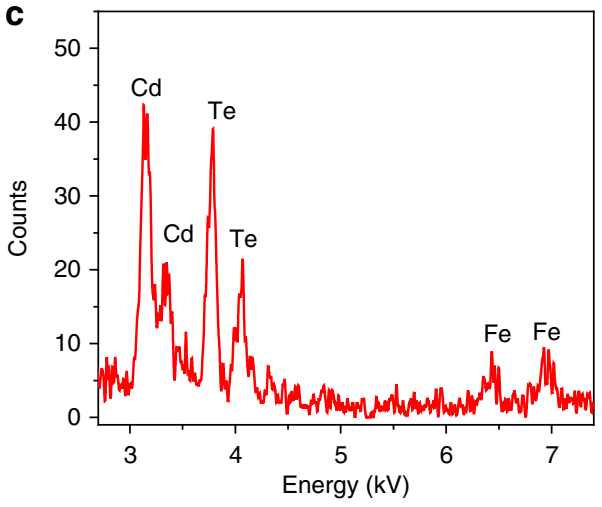

e

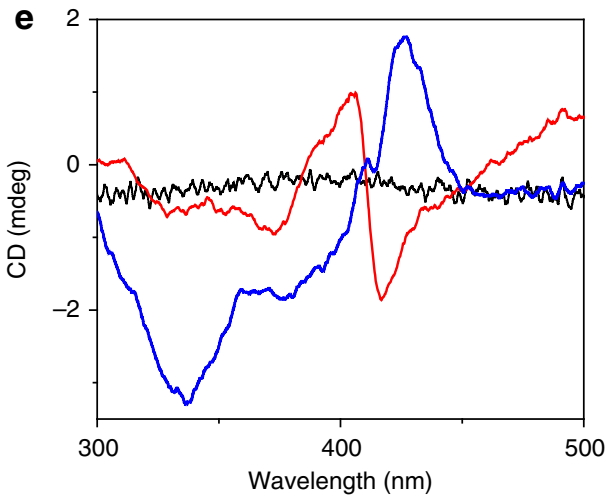

d
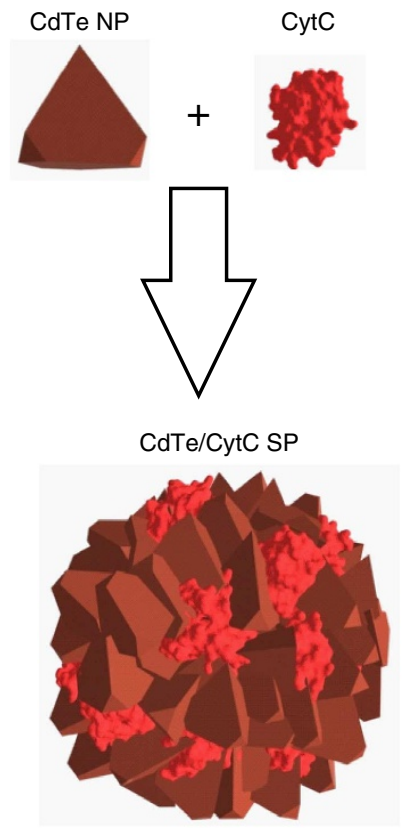

Figure 3 | Structural characterization of CdTe/CytC SPs. (a) High-resolution TEM (HR-TEM) image of the SP. Lighter areas correspond to CytC-rich phase (white-dashed circles), while the darker ones correspond to CdTe-rich phase. Scale bar, $10 \mathrm{~nm}$. (b) TEM tomographic reconstructions of CdTe/CytC SP: $X-Y$ slices ( $\mathrm{i}-\mathrm{vi}$ ) of the SP, shown in every $4.8 \mathrm{~nm}$ through the volume. 3D surface rendering(vii) and cross-section (viii) of the SP (see Supplementary Movie 1 and 2). Scale bar, $20 \mathrm{~nm}$. (c) X-ray energy dispersive spectroscopy (XEDS) spectrum for an SP in a. (d) Schematics of the formation of CdTe-CytC SPs. (e) Circular dichroisum (CD) spectra for CytC (red, $6 \mu \mathrm{M}$ ), CdTe NPs (black, $6 \mu \mathrm{M}$ ), 1:1 mixture of CdTe/CytC after 72 (blue) h.

After assembly with NPs, the proteins remain in a folded state; their denaturation would have manifested as a blue shift of the Soret band and disappearance of the peaks in $500-600 \mathrm{~nm}$ region, neither of which is observed in our system (Supplementary Fig. 7a). This conclusion is confirmed by essentially identical IR spectra of CytC before and after assembly with NPs (Supplementary Fig. 8). The circular dichroism (CD) data further substantiate that CytC molecules incorporated in SPs retain their folded, although conformationally altered, state. Original CytC molecules have negative (-) CD peaks at $208 \mathrm{~nm}$ and $220 \mathrm{~nm}$, corresponding to $\beta$-sheets and $\alpha$-helices, respectively (Supplementary Fig. 7d). In the SPs, the positive CD peak representing $\alpha$-helices at $225 \mathrm{~nm}$ dominates, and a new negative peak appears at $340 \mathrm{~nm}$ (Fig. 3e and Supplementary Fig. 7d). The conformational changes of CytC on interacting with NPs are also confirmed by examining the Soret region $\mathrm{CD}$ peaks that appear for free CytC at $408 \mathrm{~nm}(+)$ and $418 \mathrm{~nm}(-)$, typical of the haem group. The corresponding CD peaks in the assembled SPs become red-shifted and change signs (Fig. 3e), which correlates well with the change of the redox state of iron in the intact haem group. The dilution data in Supplementary Fig. 9 further confirm that these structural changes are intrinsic to SPs and are not due to intermediates or other macromolecular complexes. The observed changes in $\mathrm{CD}$, UV spectra and the conformational transitions on assembly of CytC with NPs are reminiscent of those in CytC complexes with GroEL or Cytochrome C oxidase (Supplementary Note 5) and those in concentrated solutions of globular proteins ${ }^{26}$.

Assembly mechanism. We now consider the mechanism of the formation of spherical monodisperse SPs. Since the components are similarly charged, we attribute their formation to a self-limiting process in which the electrostatic repulsion between the NPs and protein is overcome by weak attractive interactions, which corroborates previous findings (Supplementary Discussion) ${ }^{18}$. The overall attractive potential between the similarly charged CdTe $\mathrm{NPs}$ and CytC can be confirmed by calculations based on classical 
and extended Derjaguin, Landau, Verwey and Overbeek (DLVO) theory (Fig. 4a, Supplementary Methods). Although classical DLVO theory has multiple problematic points when applied to NPs, it is still instructive to show that the aggregation barrier in the pair potential is smaller than $k_{\mathrm{B}} T$. After addition of other terms, such as dipole-dipole, charge-dipole, and hydrophobic interactions (described in Supplementary Methods) the pair potential becomes attractive for all separation distances. The non-monotonic nature of these interactions and their nonadditivity at short separation distances are likely to further shift the balance towards the attractive interactions. Now the question is why such a pair potential does not lead to complete aggregation of the constituent particles.

Self-limiting processes, and therefore terminal assemblies, appear because the system evolves towards an equilibrium state as can be seen in the temporal profile of electrokinetic potential $(\zeta)$ during the assembly. Starting from $\zeta=+26 \mathrm{mV}$ and $+7 \mathrm{mV}$ corresponding to individual CdTe NPs and CytC, respectively (Supplementary Fig. 10), $\zeta$ rapidly increases from $+8 \mathrm{mV}(0 \mathrm{~h})$ to $+48 \mathrm{mV}$ within the first $5 \mathrm{~h}$. This state corresponds to the formation of large, dynamic, intermediate aggregates. Later, $\zeta$ reduces slightly to $+37 \mathrm{mV}(10 \mathrm{~h})$ and plateaus at $+31 \mathrm{mV}(70 \mathrm{~h}$, Fig. $4 \mathrm{~b})$ due to the condensation of the intermediate aggregates. The $\zeta$ values match the trend of SP diameters that peak after $\sim 1 \mathrm{~h}$ of the assembly time (see Supplementary Fig. 4).

Computer simulations are performed to reveal further details of the self-limiting assembly of the SPs. On the basis of a coarse-grained model previously developed for CdTe $\mathrm{NPs}^{3}$ (Supplementary Fig. 11a), the effective non-covalent interactions between NPs and CytC are described by the empirical 12-6 Lennard-Jones potential. The Yukawa potential models the screened charge-charge, dipole-dipole and dipole-charge interactions. As expected from previous studies, simulated tetrahedrally shaped DMAET-CdTe NPs self-assemble into flat sheets (Supplementary Fig. 11b) ${ }^{3}$. The presence of CytC markedly
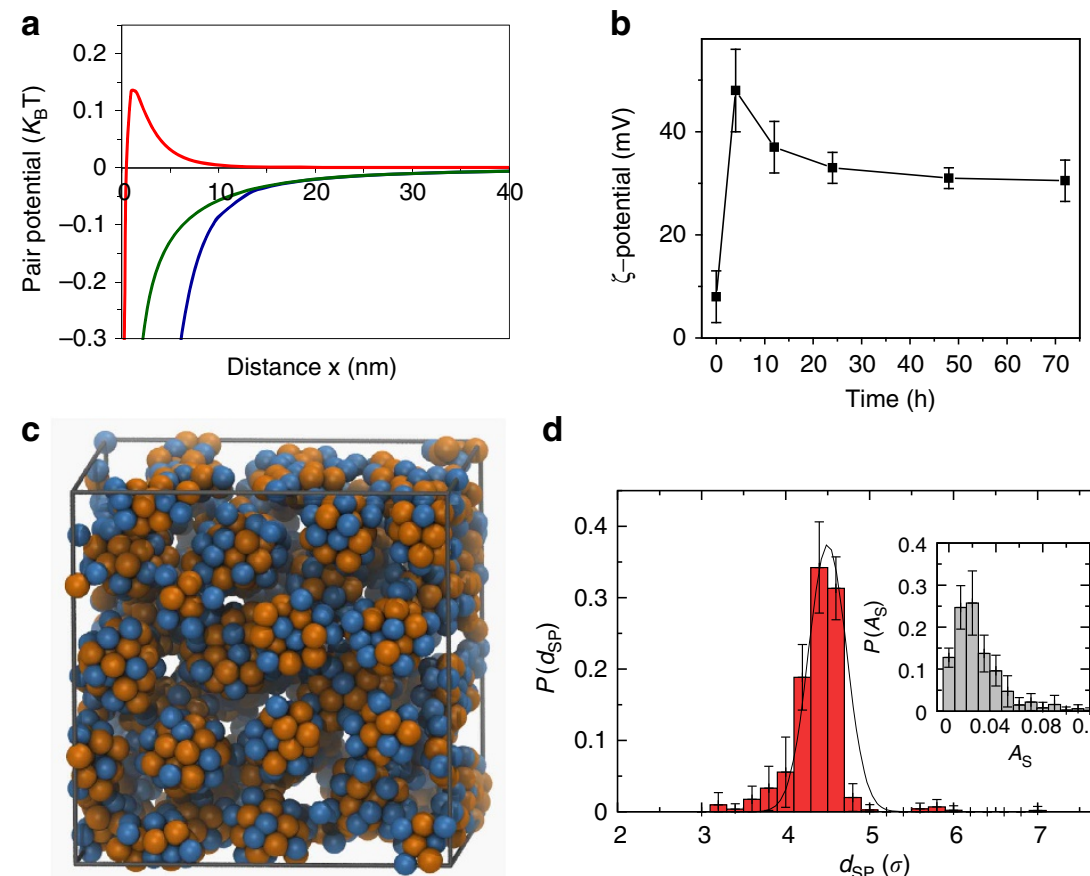

d
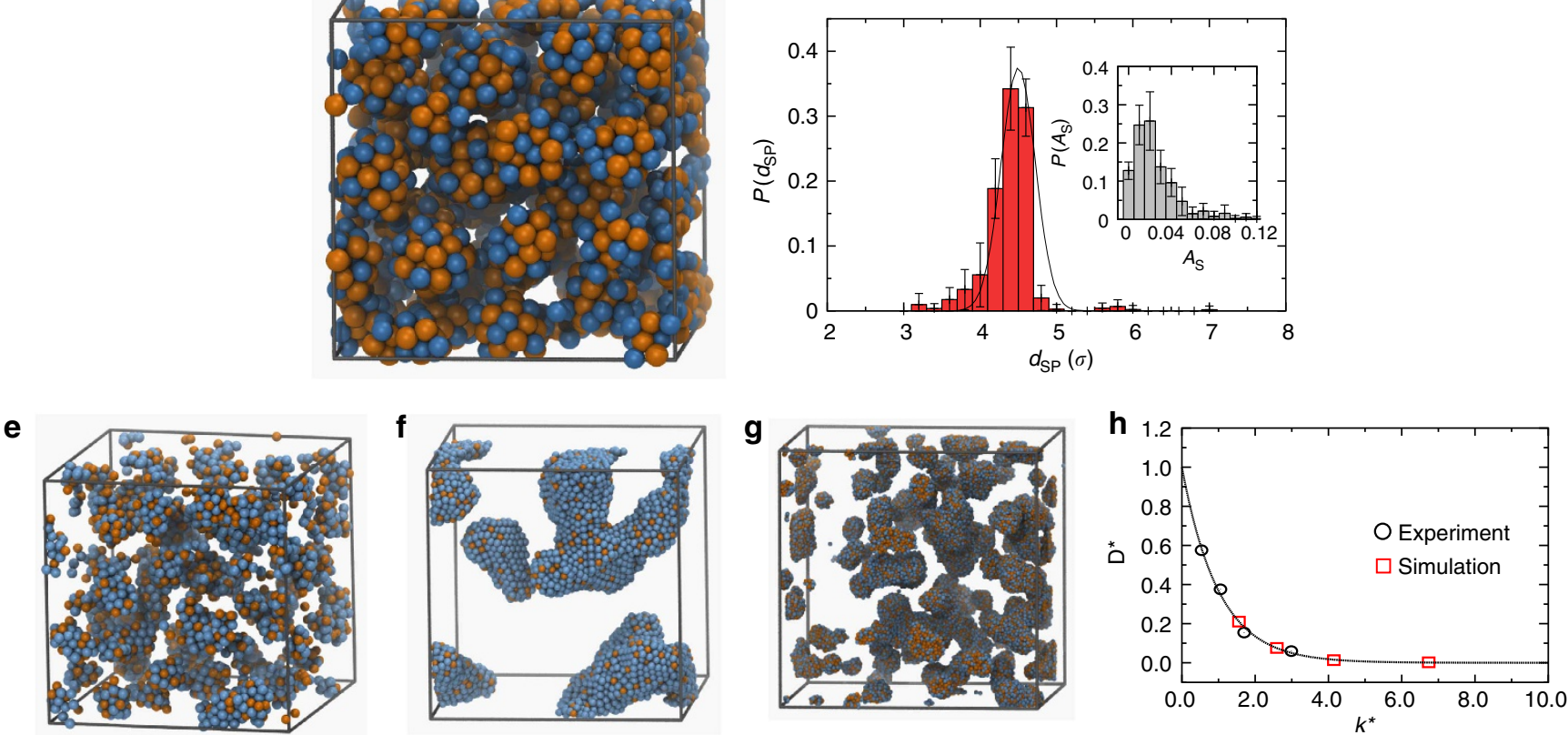

Figure 4 | Mechanism of self-assembly process. (a) Pair potential between CdTe NPs and CytC according to Derjaguin, Landau, Verwey and Overbeek (DLVO) and extended-DLVO (E-DLVO) theories: $V_{D L V O}$ (red); $V_{E-D L V O 1}$ (green); $V_{E-D L V O 2}$ (blue) (Supplementary Methods). (b) $\zeta$-potential values for the assembly of CdTe NPs with CytC at different time intervals. Error bars indicate the s.d. values from multiple measurements. (c) Spherical assemblies formed by a mixture of $1,000 \mathrm{NPs}$ (yellow) and 1,000 CytC units (blue) at $\rho \sigma^{3}=0.25$. (d) Distribution of SP sizes and of the asphericity parameter ( $A_{S}$ ) (inset) of a system composed of $8,000 \mathrm{NPs}$ and $8,000 \mathrm{CytC}$ units (Supplementary Fig. 12a). $A_{\mathrm{S}}=0$ corresponds to perfect spheres. Error bars indicate 10 equilibrated, independent configurations separated by intervals of 2,000 $\tau$. (e,f) Snapshots of mixtures with NP/CytC molar ratios of 2:1 and 6:1, respectively. (g) A snapshot of a system identical to (c), but without the inter-SP charge-charge repulsion renormalization. The images in (c,e,f and $\mathbf{g})$ were generated using the software VMD. (h) Experimental and simulated data fitted against decaying laws, that is $f(x) \sim \exp (-x)$, and plotted against dimensionless inverse screening length $\left(\kappa^{\star}\right)$ and normalized diameters $\left(D^{\star}\right)$ (see Supplementary Methods for details). 
alters their assembly pathway to produce hybrid NP-protein SPs when the attraction of CytC to the NPs is sufficiently strong. (Supplementary Fig. 11c). To facilitate the computation while keeping essential physics of the self-limiting assembly process (Supplementary Discussion), we model NPs and proteins as spheres (Fig. 4c-g). Importantly, the inter-SP repulsion strength is renormalized as the assembly progresses (Supplementary Discussion $)^{27,33,34}$ so as to describe the structural adaptation of ionic clouds around NP and CytC during SP formation. We note that the SP-SP repulsion was renormalized in previous studies, for example, in refs 34,35 , presuming that the SPs already formed. This simplified model is useful to understand the control parameters for SP assembly and to demonstrate the generality of the assembly of NPs with proteins. Despite these simplifications, this model describes our experiments remarkably well. We observe the assembly of multiple SPs for a 1:1 molar ratio of the components, while deviations from this ratio produce assemblies with irregular shapes (Fig. 4e,f). Within the SPs, NPs and CytC form small clusters of a few particles (Fig. 4c) that correspond to domains $\sim 6 \mathrm{~nm}$ in size, as observed from TEM (Fig. 3a,b). The simulated size distribution exhibits a pronounced peak (Fig. 4d) for the equilibrium state when the inter-SP repulsion balances the attraction between NPs and CytC. The small asphericity parameter (Fig. $4 \mathrm{~d}$, inset) indicates that most of the simulated aggregates are indeed spherical. Multiple SPs at their terminal size do not coalesce because the repulsion from their constituents to any approaching NP or protein exceeds the net attraction (Supplementary Fig. 12). The temporal profile of the potential energy plateaus for stable SPs, indicating the trend towards an equilibrium state (Supplementary Fig. 12b). We further observe that when the SPs reach their terminal size they become spherical in shape (Supplementary Fig. 12c,d).

Continuous normalization of the charge screening conditions reflects the continuous change in the electric double layer for NPs as they assemble. When the inter-SP charge-charge repulsion renormalization is excluded from the simulation model, the assembly is not self-limiting, leading to infinite aggregation and non-uniform SPs (Fig. 4g, and Supplementary Fig. 13) in disagreement with the experiments. This observation once again confirms that the assembly mechanism of terminal assemblies results from the balance between the net attractive forces between the NPs and CytC and their electrostatic repulsion, which should be renormalized with the SP sizes during aggregation.

The effects of important media factors such as ionic strength and temperature on the size of the terminal SPs are also captured qualitatively in our simulation model. As the salt concentration is increased, the screening length of the electrostatic repulsion decreases, leading to the reduction in the effective repulsion between freely floating NPs and CytC. As a result, the number of NPs and CytC within the terminal SPs at the terminal size shifts to lower values given that the net attraction between individual units and the repulsion renormalization are unchanged. The decrease in the SP terminal size with the increased $\mathrm{NaCl}$ concentration (Fig. 2e) is also qualitatively recovered in our simulation model (Fig. 2e, inset). The agreement between experimental and simulation data is pronounced when we fit the two data sets with decaying laws and plot them using dimensionless axes (Fig. 4h, Supplementary Methods). Meanwhile, the temperature dependence of the SP terminal size is consistent with experimental data in the lower temperature regime where $k_{B} T / \varepsilon \sim 1.0$ (Fig. 2f) with epsilon being the well depth of the Lennard-Jones potential. In this temperature range, the net effective attraction between NPs and CytC is comparable to thermal fluctuations, and the terminal SP size is fairly insensitive to temperature. For $k_{B} T / \varepsilon$ much greater than 1.0 , the attraction strength becomes weaker than thermal fluctuations, and the simulation results diverge from experimental observation with the decrease in the SP terminal size (Fig. 2g, inset). This divergence can be attributed to the CytC molecules adopting conformations other than the folded state being used in the current model.

Photoenzymatic activity. Terminal assemblies enable a simple means for fabricating complex bio-nano systems from a variety of components. They can also be called 'bionic' assemblies because they combine the properties of inorganic and biological components. A new redox enzyme, namely nitrate reductase (NRed), is incorporated as an additional component in SPs as they assemble from CytC and NPs. NRed integration into SPs leads to an increase in SP size and decrease in $\zeta$-potential while maintaining SP sphericity (Supplementary Figs 15a,b and 16). Various spectroscopy data indicated that $25 \pm 8$ NRed molecules are incorporated in each SPs; this number correlates well with the observed increase in SP diameter (Supplementary Fig. 15b-e). The positions of all peaks in the UV-Vis/CD spectra remain unchanged, indicating that the electronic state and conformation of the CytC molecules in the SPs are preserved (Supplementary Fig. 15f,g).

Nicotinamide adenine dinucleotide phosphate, (NADPH), which serves as a sacrificial electron donor, is added to the SP dispersion. Incorporation of NADPH in the SPs is observed based on XEDS data (Supplementary Fig. 15h). Direct reduction of $\mathrm{NO}_{3}^{-}$by NADPH cannot occur and must have been catalysed by NRed. We examine whether this catalytic process can be driven by light adsorption into CdTe. The direct electron transfer from CdTe to NRed; it is slow and has to be mediated by CytC. The rate of $\mathrm{NO}_{2}^{-}$production by SP-NRed in presence of NADPH increased fourfold when we illuminated the SPs at $470 \mathrm{~nm}$-the absorption peak of CdTe NPs (Fig. 5a). This indicates that the sequence of electron transfer reactions $\mathrm{NADPH} \rightarrow \mathrm{CdTe}(h v) \rightarrow$ CytC $\rightarrow \mathrm{NRed} \rightarrow \mathrm{NO}_{3}^{-}$is likely to occur. The reduced form of CytC present in SPs (Fig. 3) can initiate the process of $\mathrm{NO}_{3}^{-}$ conversion. Subsequently the photo-excited electrons from CdTe NPs are continuously supplied to CytC, which shuttles them to NRed. The holes in CdTe are scavenged by NADPH (Supplementary Note 6). Dense packing of all the organic and inorganic building blocks within SPs and the large interfacial area of the bicontinuous percolated structure of SPs facilitate all the electron transfer reactions. The importance of dense packing could also be seen for clusters of inorganic catalysts ${ }^{36}$. Side reactions, such as reduction of NP holes by oxidizable residues on the proteins, for example, tyrosine, in CytC, are nevertheless highly probable, similarly to bioconjugates ${ }^{19,36,37}$.

In the absence of light, the presence of the SPs has virtually no effect on the activity of the enzyme (Fig. 5a). Control experiments confirm the significance of the NPs and SPs for the photoenzymatic $\mathrm{NO}_{3}^{-}$reduction. These include the enzyme reaction (i) either with CdTe NPs or (ii) with CytC upon illumination (Fig. 5a, inset). None of the control experiments shows enzyme activity as high as for the illuminated SP-NRed in the presence of NADPH.

The assembled SPs remain intact after 20 min of photoreaction. However, the disassembly of SP-NRed occurred after $\sim 30 \mathrm{~min}$ of SP-NRed's exposure to light (Fig. 5c,d), which was synchronized with the drop in photoenzymatic activity (Fig. 5a, Supplementary Fig. 17). The decrease in enzymatic activity is attributed to irreversible oxidation of the NPs by the photogenerated holes. This results in removal of DMAET ligands from the surface of the NPs, and therefore the loss of attractive intermolecular interactions. The synchronization of the drop in enzymatic reduction and onset of disassembly vividly demonstrates that the formation of the terminal SPs is necessary to obtain higher photoenzymatic 

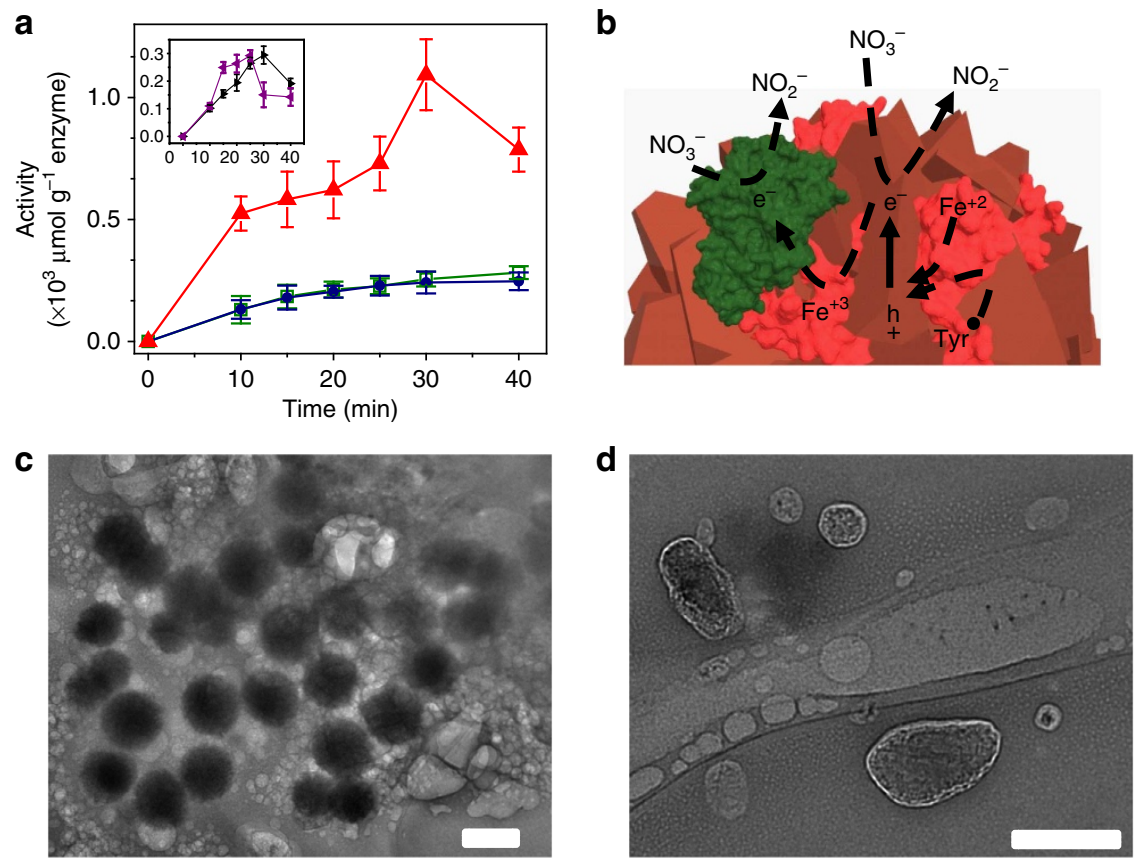

Figure 5 | Functional integration of SPs with photoenzymatic activity. (a) Formation of nitrite for SP-nicotinamide adenine dinucleotide phosphate (NADPH)-nitrate reductase (NRed) (red) excited at $470 \mathrm{~nm}$ and for NADPH-NRed (green) and SP-NADPH-NRed in dark (blue). Inset: formation of nitrite for NADPH-NRed being excited at $470 \mathrm{~nm}$ in presence of only one of SP components: either CdTe NPs (black) or CytC (purple) when no hybrid SPs were formed. Errors bars are based on triplicate experimental measurements. (b) Schematics of the reactions on the photoexcitation of SP-NADPH-NRed. Nitrate reductase is represented as a surface model from PyMol software, PDB entry is 2BIH. TEM images of (c) SP-NADPH-NRed after 20 min and (d) after $40 \mathrm{~min}$ of the photoenzymatic reaction. Scale bar, $100 \mathrm{~nm}$.

activity. TEM images of SPs after extended photoreaction support this conclusion (Fig. 5d).

\section{Discussion}

In summary, CdTe NPs and like-charged proteins self-organize into self-limiting SPs, following a pattern previously unseen for the individual components. These terminal superstructures display complex internal organization and can incorporate multiple biological components. We also demonstrate that the protein component retains its functionality. 'Bionic' integration of the light absorbing properties of the NPs and the catalytic properties of the enzyme was achieved. Since only generic attractive-repulsive forces are required for formation of the terminal SPs, we expect that they can be made from a large variety of NPs and proteins. The simplicity of their preparation is conducive to a large variety of such structures that can form in the laboratory and in nature. We stress that this simplicity of preparation and seemingly good match with theoretical and computational description of the attraction-repulsion balance do not imply the simplicity of the interactions between CdTe and $\mathrm{CytC}$ as well as other proteins/NPs. The altered conformation of the protein is a clear indication of their complexity and nonmonotonic nature of such interactions associated with the 'softness' of the interacting particles. Consideration of these details in subsequent theoretical and computational treatments will allow provide prediction of explicit NP interaction and effective integration of inorganic and biocatalytic properties in SP assemblies.

\section{Methods}

Synthesis of DMAET-stabilized CdTe NPs. DMAET-CdTe NPs were prepared following the previous study ${ }^{22}$. In brief, $\sim 0.7 \mathrm{~g}$ of $\mathrm{Cd}\left(\mathrm{ClO}_{4}\right)_{2} \cdot 6 \mathrm{H}_{2} \mathrm{O}$ (Alfa Aesar) dissolved in $200 \mathrm{ml}$ of water was mixed with $\sim 0.45 \mathrm{~g}$ of DMAET (Sigma-Aldrich). Subsequently, pH of the medium was adjusted to be $\sim 5.5$ by addition of $0.1 \mathrm{M} \mathrm{HCl}$ solution. The reaction mixture was placed in three $250 \mathrm{ml}$ neck round bottom flask with a septum, valve and condenser, and was bubbled with $\mathrm{N}_{2}$ gas for at least $30 \mathrm{~min} . \mathrm{H}_{2} \mathrm{Te}$ gas formed by reacting $\sim 0.2 \mathrm{~g}$ of $\mathrm{Al}_{2} \mathrm{Te}_{3}$ (Materion) with $0.5 \mathrm{M}-\mathrm{H}_{2} \mathrm{SO}_{4}$ was passed through the reaction mixture for $10 \mathrm{~min}$ at room temperature. Then, the reaction proceeded for $1-1.5 \mathrm{~h}$ under magnetic stirring at $100^{\circ} \mathrm{C}$. The reaction was stopped by cooling down the reactor at room temperature for $\sim 30 \mathrm{~min}$. The average diameter of the synthesized NPs was $3.8 \pm 0.4 \mathrm{~nm}$. The reaction time varied to obtain different sizes of the NPs.

Assembly of CdTe/CytC SPs. Typically, $1 \mathrm{ml}$ of NP dispersion containing $6 \mu \mathrm{M}$ of NPs was precipitated at $4^{\circ} \mathrm{C}$ for $24 \mathrm{~h}$. Subsequently, the dispersion was centrifuged at 6,000 r.p.m. for $20 \mathrm{~min}$ at $15^{\circ} \mathrm{C}$. The supernatant was discarded, and the NPs were redispersed in $1 \mathrm{ml}$ of water at $\mathrm{pH} 5$ (adjusted by the addition of $0.1 \mathrm{M} \mathrm{HCl}$ ). Finally, the NP dispersion was mixed with $20 \mu \mathrm{l}$ of $300 \mu \mathrm{M}$-CytC aqueous solution and was stored for $72 \mathrm{~h}$. The assembly medium contained desired concentrations $(0.1,0.5,1.0,2.0 \mathrm{M})$ of $\mathrm{NaCl}$, or it was stored at different temperatures $\left(4,20,35,55,70^{\circ} \mathrm{C}\right)$. Typically, samples for TEM/SEM studies were diluted before imaging.

Enzymatic reduction of nitrate. Ten microlitres of enzyme, NRed, solution (Cayman Chemical) was added to $770 \mu \mathrm{l}$ of aqueous solution containing $\mathrm{CdTe} /$ CytC SP $(40 \mu \mathrm{l})$, cofactor (NADPH, $10 \mu \mathrm{l}$, Cayman Chemical) and $\mathrm{NaNO}_{3}$ $(150 \mu \mathrm{l}, 200 \mathrm{mM})$. The solution was placed in in $800 \mu \mathrm{l}$ quartz cuvette (Starna cells) and illuminated at $470 \mathrm{~nm}$ via Horiba FluoroMax-3 monochromator for $1 \mathrm{~h}$ with slit width for excitation at $20 \mathrm{~nm}$. The wavelength matched to the onset of excitonic absorption of CdTe NPs in the bionic SPs. During illumination, $30 \mu \mathrm{l}$ of aliquots diluted with $470 \mu \mathrm{l}$ of water at desired time intervals were taken for analysis.

Nitrate reduction assay. Before the assay, each of $500 \mu \mathrm{l}$ of diluted samples was mixed with $50 \mu \mathrm{l}$ of 2,3-diaminonaphthalene (DAN) and $100 \mu \mathrm{l}$ of $2.8 \mathrm{M}-\mathrm{NaOH}$ solution (Cayman Chemical). Fluorescence emission at $417 \mathrm{~nm}$ of the samples was recorded by exciting the samples at $375 \mathrm{~nm}$ (Synergy 2 plate reader, Biotek). The activity was measured by a standard fluorescence assay method to quantify produced $\mathrm{NO}_{2}^{-}$( $\mu \mathrm{molg}{ }^{-1}$-enzyme) (https://www.caymanchem.com/pdfs/ 780051.pdf.). The concentration of $\mathrm{NO}_{2}^{-}$was determined by a standard plot of different concentrations of $\mathrm{NO}_{2}^{-}$(see Supplementary Fig 18). 


\section{References}

1. Tang, Z. Y., Kotov, N. A. \& Giersig, M. Spontaneous organization of single CdTe nanoparticles into luminescent nanowires. Science 297, 237-240 (2002).

2. Lin, Y. et al. Ultrathin cross-linked nanoparticle membranes. J. Am. Chem. Soc. 125, 12690-12691 (2003)

3. Tang, Z. Y., Zhang, Z. L., Wang, Y., Glotzer, S. C. \& Kotov, N. A. Self-assembly of CdTe nanocrystals into free-floating sheets. Science 314, 274-278 (2006).

4. Srivastava, S. et al. Light-controlled self-assembly of semiconductor nanoparticles into twisted ribbons. Science 327, 1355-1359 (2010).

5. Ishii, D. \& Kinbara, K. et al. Chaperonin-mediated stabilization and ATPtriggered release of semiconductor nanoparticles. Nature 423, 628 (2003).

6. Kotov, N. A. Inorganic nanoparticles as protein mimics. Science 330, 188-189 (2010).

7. Kostiainen, M. A. et al. Electrostatic assembly of binary nanoparticle superlattices using protein cages. Nat. Nanotechnol. 8, 52-56 (2013).

8. Rajendran, V., Konig, A., Rabe, K. S. \& Niemeyer, C. M. Photocatalytic activity of protein-conjugated CdS nanoparticles. Small 6, 2035-2040 (2010).

9. Warner, M. G. \& Hutchison, J. E. Linear assemblies of nanoparticles electrostatically organized on DNA scaffolds. Nat. Mater. 2, 272-277 (2003).

10. Dabbousi, B. O., Murray, C. B., Rubner, M. F. \& Bawendi, M. G. LangmuirBlodgett Manipulation of Size-Selected CdSe Nanocrystallites. Chem. Mater. 6, 216-219 (1994).

11. Bruchez, M., Moronne, M., Gin, P., Weiss, S. \& Alivisatos, A. P. Semiconductor nanocrystals as fluorescent biological labels. Science 281, 2013-2016 (1998).

12. Bayraktar, H., You, C. C., Rotello, V. M. \& Knapp, M. J. Facial control of nanoparticle binding to cytochrome c. J. Am. Chem. Soc. 129, 2732 (2007)

13. You, C.-C. et al. Detection and identification of proteins using nanoparticlefluorescent polymer 'chemical nose' sensors. Nat. Nanotech. 2, 318-323 (2007).

14. Lundqvist, M. et al. Nanoparticle size and surface properties determine the protein corona with possible implications for biological impacts. PNAS 105 , 14265-14270 (2008).

15. Miller, M. A. et al. Antibody nanoparticle dispersions formed with mixtures of crowding molecules retain activity and in vivo bioavailability. J. Pharm. Sci. 101, 3763-3778.

16. Murthy, A. K. et al. Charged gold nanoparticles with essentially zero serum protein adsorption in undiluted fetal bovine serum. J. Am. Chem. Soc. 135, 7799-7802.

17. Hochella, M. F. et al. Nanominerals, mineral nanoparticles, and Earth systems. Science 319, 1631-1635 (2008).

18. Xia, Y. S. et al. Self-assembly of self-limiting monodisperse supraparticles from polydisperse nanoparticles. Nat. Nanotechnol. 6, 580-587 (2011).

19. Katz, E., Zayats, M., Willner, I. \& Lisdat, F. Controlling the direction of photocurrents by means of CdS nanoparticles and cytochrome c-mediated biocatalytic cascades. Chem. Commun. 1395-1397 (2006).

20. Ipe, B. I. \& Niemeyer, C. M. Nanohybrids composed of quantum dots and cytochrome P450 as photocatalysts. Angew. Chem. Int. Ed. 45, 504-507 (2006).

21. Ge, J., Lei, J. D. \& Zare, R. N. Protein-inorganic hybrid nanoflowers. Nat. Nanotechnol. 7, 428-432 (2012).

22. Gaponik, N. et al. Thiol-capping of CdTe nanocrystals: An alternative to organometallic synthetic routes. J. Phys. Chem. B 106, 7177-7185 (2002).

23. Haas, A. S. et al. Cytochrome $c$ and cytochrome $c$ oxidase: Monolayer assemblies and catalysis. J. Phys. Chem. B 105, 11351-11362 (2001).

24. Koppenol, W. H., Rush, J. D., Mills, J. D. \& Margoliash, E. The Dipole-moment of cytochrome-C. Mol. Biol. Evol. 8, 545-558 (1991).

25. Zheng, J. W. et al. Two-dimensional nanoparticle arrays show the organizational power of robust DNA motifs. Nano Lett. 6, 1502-1504 (2006).

26. Cheung, J. K., Shen, V. K., Errington, J. R. \& Truskett, T. M. Coarse-grained strategy for modeling protein stability in concentrated solutions. III: Directional protein interactions. Biophys. J. 92, 4316-4324 (2007).

27. Sciortino, F., Mossa, S., Zaccarelli, E. \& Tartaglia, P. Equilibrium cluster phases and low-density arrested disordered states: The role of short-range attraction and long-range repulsion. Phys. Rev. Lett. 93, 055701 (2004).

28. Ramsden, J.J. The stability of superspheres. Proc. R. Soc. Lond. A 413, 407-414 (1987).
29. Zeleny, J. The electrical discharge from liquids points and a hydrostatic method of measuring the electric intensity at their surfaces. Phys. Rev. 3, 69-91 (1914).

30. Kittler, S. et al. The influence of proteins on the dispersability and cellbiological activity of silver nanoparticles. J. Mater. Chem. 20, 512-518 (2010).

31. Kim, Y. et al. Stretchable nanoparticle conductors with self-organized conductive pathways. Nature 500, 59-64 (2013).

32. Andrade, A. A. et al. Two-photon absorption investigation in reduced and oxidized cytochrome c solutions. Chem. Phys. Lett. 390, 506-510 (2004).

33. Kung, W., Gonzalez-Mozuelos, P. \& Olvera de la Cruz, M. A minimal model of nanoparticle crystallization in polar solvents via steric effects. J. Chem. Phys. 133, 074704 (2010)

34. Zhang, D., Gonzalez-Mozuelos, P. \& Olvera de la Cruz, M. Cluster formation by charged nanoparticles on a surface in aqueous solution. J. Phys. Chem. C 114, 3754-3762 (2010)

35. Israelachvili, J. N. Intermolecular and Surface Forces 3rd edn (Academic Press, 2011).

36. Slocik, J. M., Govorov, A. O. \& Naik, R. R. Photoactivated biotemplated nanoparticles as an enzyme mimic. Angew. Chem. Int. Ed. 47, 5335-5339 (2008).

37. Gerhards, C., Schulz-Drost, C., Sgobba, V. \& Guldi, D. M. Conjugating luminescent CdTe quantum dots with biomolecules. J. Phys. Chem. B 112, 14482-14491 (2008).

\section{Acknowledgements}

This material is based on work partially supported by the Center for Solar and Therma Energy Conversion, an Energy Frontier Research Center funded by the U.S. Department of Energy, Office of Science, Office of Basic Energy Sciences under Award Number \#DESC0000957. This work was supported by the Center for Photonic and Multiscale Nanomaterials (C-PHOM) funded by the National Science Foundation Materials Research Science and Engineering Center program DMR 1120923. We acknowledge support from NSF under grants ECS-0601345; EFRI-BSBA 0938019; CBET 0933384; CBET 0932823; and CBET 1036672. This material is based on work supported by, or in part by, the U.S. Army Research Office under Grant Award No. W911NF-10-1-0518. 'This material is based on work supported by the DOD/ASDRE under Award No. N00244-09-1-0062. Any opinions, findings and conclusions or recommendations expressed in this publication are those of the author(s) and do not necessarily reflect the views of the DOD/ASDRE. The support from NIH grant GM085043 (P.Z.) is gratefully acknowledged. We thank the University of Michigan's EMAL for its assistance with electron microscopy, and the NSF grant \#DMR-9871177 for funding of the JEOL 2010F analytical electron microscope used in this work.

\section{Author contributions}

J.I.P, S.S., G.Q.S and J.H.B performed experimental work on synthesis and studies of SPs. T.D.N. performed computer simulations. K.S. performed transmission electron microscopy for this study. G.Z. and P.Z. carried out 3D TEM tomography study. T.D.N. and S.C.G. planned the simulation study and analysed the simulation data. N.A.K conceived this work and analysed the data. J.I.P, T.D.N, S.S., S.C.G and N.A.K. cowrote the manuscript.

\section{Additional information}

Supplementary Information accompanies this paper at http://www.nature.com/ naturecommunication

Competing financial interests: The authors declare no competing financial interests.

Reprints and permission information is available online at http://npg.nature.com/ reprintsandpermissions/

How to cite this article: Park, J. I. et al. Terminal supraparticle assemblies from similarly charged protein molecules and nanoparticles. Nat. Commun. 5:3593 doi: $10.1038 /$ ncomms4593 (2014). 